\title{
Medical conditions and outcomes after spinal cord injury
}

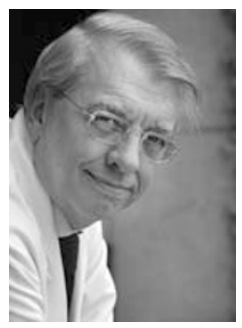

JJ Wyndaele, Editor

Antwerp University Hospital, Antwerp, Belgium

E-mail: spinalcord@uza.be

Dear Spinal Cord reader,

Before discussing the scientific content of this Spinal Cord issue, I want to let you know that I recently received a book entitled 'To be a neurosurgeon, a memoir' written by the former Editorin-Chief of Spinal Cord, Phillip Harris. ${ }^{*}$ The book is not strictly a scientific work, but a personal account of 87 years pursuit of excellence during a period of rapid technical advance in neurosurgery. It is no conventional memoir but, as written in the prefix, is designed to appeal to his family, medics and a lay audience. It is a pleasant book to read, deals with interesting historical and personal data and describes meetings with many of the great figures of the related disciplines. Through its content and the way it is presented, the book will be enjoyable reading for members of ISCOS and a broader readership.

In this June issue, several manuscripts deal with medical conditions and outcomes in individuals with spinal cord pathology.

Hill et al. performed a systematic literature review on measurement properties of quality of life (QOL) outcome measures. They found that the WHOQOL-BREF was the most acceptable and established instrument to assess quality of life after spinal cord injury at present. The SIP68, QOLP-PD, SF-36 V and SWBI are promising, but require further evaluation of their measurement properties.

Two overall interesting papers, part of the Stockholm Thessaloniki Acute Traumatic Spinal Cord Injury Study (STATSCIS), by Divanoglou et al. characterized patient populations and compared acute management after traumatic Spinal Cord Injury (TSCI). Despite largely similar core clinical characteristics, the Thessaloniki and Stockholm cases underwent significantly different acute management. There were large discrepancies in terms of one-year outcome and complications. As the main difference between regions was the presence or absence of a SCI system of care, rather than differences in availability of modern medicine, the mere presence of the latter does not seem to be sufficient to guarantee adequate outcomes. This study provides strong evidence that a SCI system of care should be implemented urgently in Greece. The conclusion is strong and applicable to many regions in the world. It contradicts the opinion sometimes expressed that well structured spinal care is no longer needed in regions where it has existed for decades. Another contribution deals with the burden of SCI in Iran (Rahimi-Movagha $\mathrm{r}$ et al.).

Several contributions deal with practical clinical items: the use of pulsatile bolus infusion of intrathecal baclofen to reverse the need for increasing dosages in patients with probable tolerance (Heetla et al.); a review suggesting that cranberry may not be effective at preventing urinary tract infections in SCI individuals (Opperman); evaluation of the SCI application of the International Classification of External Cause of Injury (ICECI) (Lee et al.); factors related to time to first job (Krause et al.); and stepwise protocol for bowel management (Coggrave and Norton). Finally there are two manuscripts on animal studies: changes of pubococcygeus muscle tension (Lu et al.) and transplantation of a combination of autologous neural differentiated and undifferentiated mesenchymal stem cells (Pedram et al.).

*To be a neurosurgeon, A memoir, Phillip Harris, The Memoir Club Durham 2009, ISBN 978-1-84104-137-7. Profits from the sale go to research into 50\% Epilepsy and 50\% Spinal disorders. 